\title{
The IL-33 receptor (ST2) regulates early IL-13 production in fungus-induced allergic airway inflammation
}

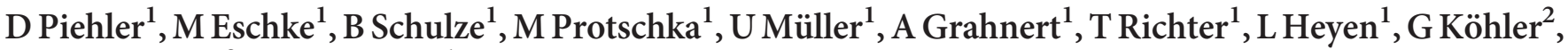 \\ F Brombacher ${ }^{3}$ and $G$ Alber ${ }^{1}$
}

\begin{abstract}
Allergic airway inflammation (AAl) in response to environmental antigens is an increasing medical problem, especially in the Western world. Type 2 interleukins (IL) are central in the pathological response but their importance and cellular source(s) often rely on the particular allergen. Here, we highlight the cellular sources and regulation of the prototypic type 2 cytokine, IL-13, during the establishment of AAI in a fungal infection model using Cryptococcus neoformans. IL-13 reporter mice revealed a rapid onset of IL-13 competence within innate lymphoid cells type 2 (ILC2) and IL-33R ${ }^{+}$Thelper (Th) cells. ILC2 showed IL-33-dependent proliferation upon infection and significant IL-13 production. Th cells essentially required IL-33 to become either GATA3 ${ }^{+}$or GATA3 $^{+} /$Foxp $^{+}{ }^{+}$hybrids. GATA3 ${ }^{+}$Th cells almost exclusively contributed to IL-13 production but hybrid GATA3 ${ }^{+} /$Foxp $^{+}{ }^{+}$Th cells did not. In addition, alveolar macrophages upregulated the IL-33R and subsequently acquired a phenotype of alternative activation $\left(\mathrm{Ym}^{+}{ }^{+}, \mathrm{FIZZ1}^{+}\right.$, and arginase $-1^{+}$) linked to type 2 immunity. Absence of adaptive immunity in rag $2^{-1-}$ mice resulted in attenuated AAl, revealing the need for Th2 cells for full AAI development. Taken together, in pulmonary cryptococcosis ILC2 and GATA $^{+}$Th2 cells produce early IL-13 largely IL-33R-dependent, thereby promoting goblet cell metaplasia, pulmonary eosinophilia, and alternative activation of alveolar macrophages.
\end{abstract}

\section{INTRODUCTION}

Allergic airway inflammation (AAI) is a growing medical problem that is generally associated with type 2 inflammation of the lower airways. ${ }^{1}$ Depending on disease severity, AAI is characterized by sneezing, coughing, bronchial obstruction by mucous plugs, and type 1 hypersensitivity. In addition, exacerbations of asthma can result in sudden death of affected individuals. $^{2,3}$ Development of AAI can be enforced by different cell populations in combination with soluble mediators that activate immune cells and non-hematopoietic cells subsequently, inducing cellular recruitment to affected tissues such as the lung. ${ }^{2,4,5}$ Several classes of antigens such as microbes, arthropod products or plant pollen have been shown to induce AAI with underlying uncontrolled type 2 immunity. ${ }^{2}$ Recently, it has been taken into account that several fungal species are involved in various diseases of the airways that are type 2 biased, e.g., allergic fungal rhinosinusitis ${ }^{6,7}$ or allergic bronchopulmonary aspergillosis. ${ }^{8}$ Type 2 cytokines have an extraordinary role with interleukin (IL)-4, IL-5, and IL-13 being the most prominent cytokines that are already used as therapeutic targets in humans. ${ }^{9}$ As different routes can induce type 2 immunity, especially in the airways, it is necessary to distinguish antigen classes, e.g., model allergens and fungal pathogens. Pulmonary infection with Cryptococcus neoformans, an ubiquitous fungal pathogen, is associated with type 2 immunity in experimental mouse and rat models of AAI development. ${ }^{10,11}$ In addition, there is support for C. neoformans-dependent development of AAI in humans as well. ${ }^{12,13}$ In previous studies that focused on late time points of fungal infection, we already demonstrated the importance of IL-13 using IL-13 $-1-$ and IL-13 ${ }^{\text {tg/ }+}$ mice. ${ }^{14}$ IL-13 has been object of several studies regarding experimental pulmonary

${ }^{1}$ Institute of Immunology/Molecular Pathogenesis, Center for Biotechnology and Biomedicine, College of Veterinary Medicine, University of Leipzig, Leipzig, Germany. ${ }^{2}$ Institute of Pathology, Klinikum Fulda gAG, Fulda, Germany and ${ }^{3}$ International Center for Genetic Engineering and Biotechnology, Cape Town Component \& SAMRC, UCT, IDM, Division of Immunology, Cape Town, South Africa. Correspondence: D Piehler (piehler@rz.uni-leipzig.de) 
cryptococcosis. $^{14-19}$ IL-13 ${ }^{-1-}$ mice and other strains with compromised IL-13 signaling showed a significant reduction of alternative macrophage activation, almost absent mucus induction in airway epithelium, reduced airway hyperreactivity, and attenuated eosinophil recruitment placing IL-13 in the center of immunopathology observed in chronic pulmonary cryptococcosis that closely resembles AAI. ${ }^{14}$ However, early sources of IL-13 have not been identified in this pulmonary infection, although early IL-13-producing cells would offer unique targets for combined and specific treatments. Since AAI-associated remodeling of the airways is not reversible in most cases and requires precocious interventional therapy, an early therapeutic strategy is particularly attractive. Although it is undoubtedly clear that IL-13 has a central role in nonprotective immune responses and concomitant AAI development, only a single study investigated the potential source(s) of IL-13 during early C. grubii H99 infection emphasizing a role of the IL-33 receptor in IL-13 induction. ${ }^{14}$ However, in this study, a serotype A strain (i.e., H99) was used that is preferentially found in North America and shows rapid pulmonary proliferation and dissemination largely irrespective of major type 2 cytokines, IL- 4 and IL-13. ${ }^{15}$ In contrast, serotype D is more prevalent in Europe and the clinical isolate 1841 used in our study depends non-redundantly on type 2 immunity to cause AAI. ${ }^{20}$ Thus, the study presented here represents an exquisite system to unravel mechanisms of fungus-induced type 2 cytokine-dependent AAI.

We show that during early cryptococcal infection innate lymphoid cells type 2 (ILC2) and GATA3 ${ }^{+}$T helper (Th) 2 cells are the IL-13-producing cells that are responsible for the hallmarks of AAI by mediating eosinophil recruitment, alternative macrophage activation shown by Ym1, FIZZ1, arginase-1 production, and goblet cell metaplasia. Infection also drives the development of previously unrecognized GATA3 $^{+} /$Foxp $^{+}$Th cells in cryptococcosis that do not contribute to IL-13 production. We also demonstrate that these processes are dominantly driven by the expression of the IL33R receptor on ILC2, Th2 cells, and alveolar macrophages. In addition, the development of type 2 immunity in this model of fungal sensitization requires adaptive immunity as demonstrated in rag2-deficient mice.

\section{RESULTS}

\section{ILC2 and Th cells are the sources of early IL-13}

To analyze the onset of pulmonary IL-13 production during early infection in Balb/c mice, we determined IL-13 production within the first 3 weeks following intranasal infection (Figure 1a). IL-13 production was increased 2-3 weeks after infection concomitantly with pulmonary mucus production (confirmed by periodic acid-Schiff (PAS) score and gob5 expression ${ }^{21}$ ) and eosinophilic inflammation already 14 days post infection (Dpi; Figure 1b) revealing early immunopathology in the lungs of infected mice. Interestingly, increasing IL-13 production and subsequent immunopathology was not associated with increased fungal burden as our strain C. neoformans 1841 showed a steady-state lung burden within the first 3 weeks after infection (Figure 1c).

In order to identify cellular sources of IL-13 production, IL-13-enhanced green flourescence protein (eGFP) reporter $\mathrm{Balb} / \mathrm{c}$ mice $\left(i l 13^{e G F P /+}\right)^{22}$ were infected and IL-13 was monitored by FACS (Figure 2a). In accordance with incremental IL-13 secretion by pulmonary cells (Figure 1a), we found an increasing frequency of $\mathrm{IL}^{-13 e G F P}{ }^{+}$cells (Figure 2a), which was positive for the activation marker ICOS (Supplementary Figure S1 online). The surface staining revealed two distinguishable cell populations with one being $\mathrm{CD} 4{ }^{+}$Th cells predominantly expressing the receptor for IL-33 (ST2, in this manuscript termed IL-33R; Figure 2b, gate, upper left plot), and CD4-negative cells, also expressing IL-33R (Figure 2b, gate, lower left plot), CD90.2, ICOS, and CD25, but no other conventional lineage antigens, meeting the recent definition of ILC2. ${ }^{23}$ Interestingly, even under naive conditions a higher percentage of ILC2 revealed to be $\mathrm{eGFP}^{+}$compared with Th cells (Figure 2b, right panel, naive). Upon infection, ILC2 also showed superior induction of eGFP indicating a greater potential to produce IL-13 compared with Th cells on a per-cell basis (Figure $\mathbf{2 b}, \mathbf{c}$ ). This demonstrates that during infection with $C$. neoformans, IL-13 is mainly produced by IL-33R ${ }^{+}$Th cells and ILC2.

\section{Ablation of activating receptors, IL-33R and IL-25R, reduces ILC2 proliferation and cytokine synthesis}

Next, we investigated cytokines that can induce proliferation as well as strong cytokine production in infected lung tissue. ${ }^{24-26}$ Interestingly, we found selective and time-dependent induction of pulmonary expression of $i l 33$, but not $i l 25$ or $t s l p$, upon infection with C. neoformans (Figure 3a). Increased il33 mRNA expression was associated with elevated total numbers of ILC2 and increased Ki67 staining (Figure 3b). This was specific for the lung, as no significant increase of ILC2 could be observed in the draining lymph nodes or the spleen, even up to 6 weeks after infection (data not shown). Therefore, we focused on the analysis of pulmonary ILC2. Importantly, comparison of ILC2 phenotype based on CD25, CD90.2, ICOS, GATA3, and $\mathrm{KLRG}^{27}$ revealed no differences between wild-type (wt), $s t 2^{-1-}$, and il17rb/st $2^{-1-}$ mice (Figure 3c). This phenotypic characterization allowed (without the use of IL-33R antibody) the identification of ILC2 in st2 ${ }^{-1-}$ and $i l 17 \mathrm{rb} / \mathrm{st} 2^{-1-}$ mice comparable to wt $\left(s t 2^{+/+}\right)$mice shown in Figure $\mathbf{2 b}$ and Supplementary Figure S2. Previously, it had been shown that $i l 17 \mathrm{rb} / \mathrm{st} 2^{-1-}$ mice showed almost total blockade of ILC2 proliferation in a gastrointestinal parasite model using Nippostrongylus brasiliensis, demonstrating the essential role of the IL-25/IL-33 pathways in ILC2 activation. ${ }^{22}$ We confirmed this in C. neoformans infection, thereby uncovering a dominant role for IL-33 as $s t 2^{-1-}$ and illirb/st $2^{-1-}$ mice showed similar histopathological alterations, especially a strong reduction in the number of mucus-producing epithelial cells after infection compared with wt animals (Figure 4). $s t 2^{-1-}$ and $i l 17 \mathrm{rb} / \mathrm{st} 2^{-1-}$ mice showed similarly reduced IL-5, IL-13, and IL-17A production after infection compared with wt 


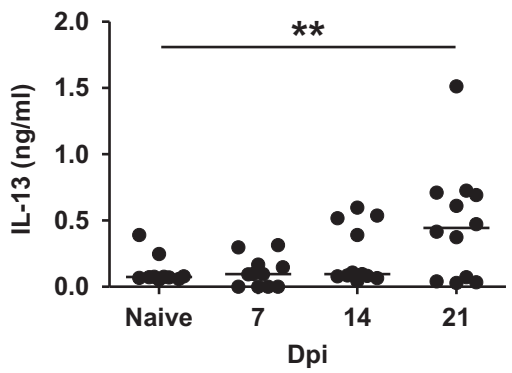

b
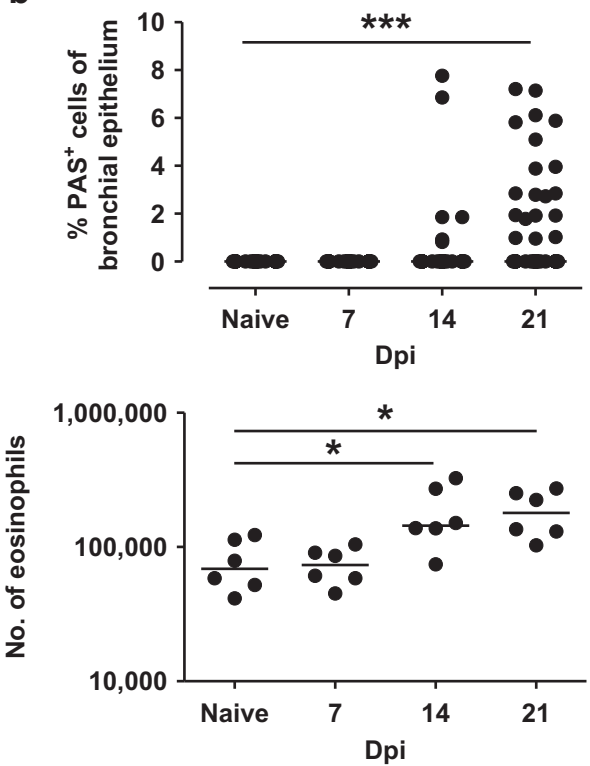
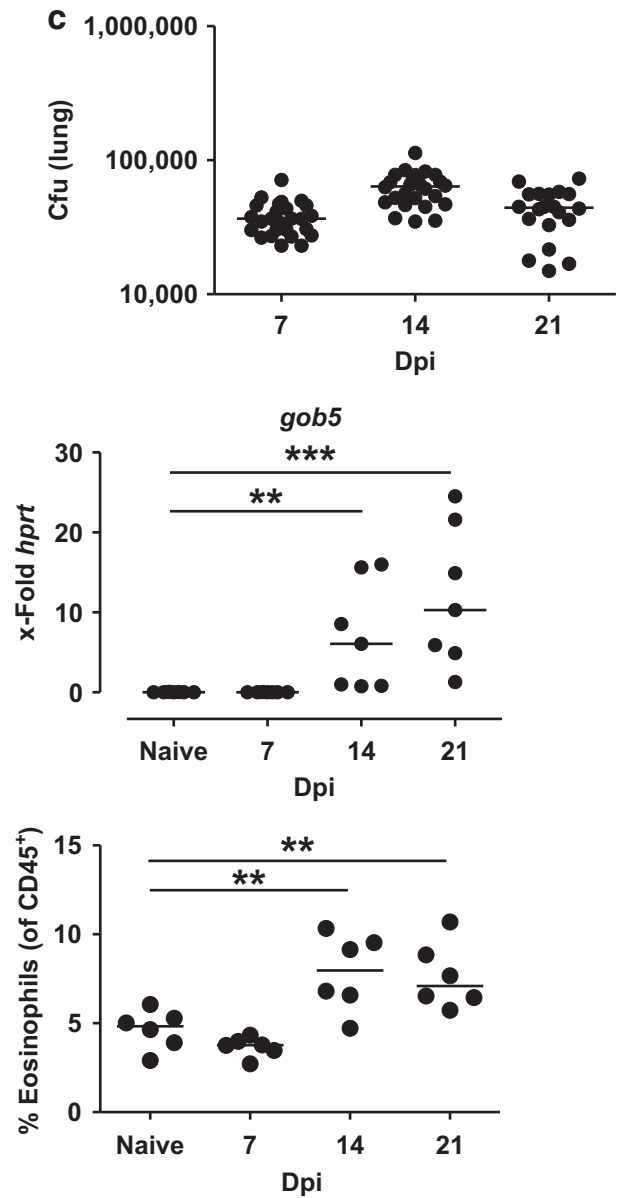

Figure 1 Early interleukin (IL)-13 secretion and mucus production is independent of fungal burden. IL-13-enhanced green flourescence protein (eGFP) reporter mice were infected intranasally with $C$. neoformans 1841 and analyzed at indicated time points $(n=5-7$ mice/experiment). (a) IL-13 ELISA of supernatants of pulmonary leukocytes restimulated with ionomycin and phorbol 12-myristate 13-acetate (PMA) for $4 \mathrm{~h}$ (individual mice). Data from two independent experiments were pooled. One-way analysis of variance (ANOVA) with Dunnett's multiple comparison test was performed with ${ }^{* \star} P \leqslant 0.01$ (b) PAS score was calculated from counting PAS ${ }^{+}$epithelial cells of ten bronchi of each mouse and gob5 (ref. 21) expression was analyzed by quantitative PCR (as described in materials and methods section). Kruskal-Wallis test with Dunn's multiple comparison test was performed with ${ }^{\star \star} P \leqslant 0.01 ;{ }^{* \star *} P \leqslant 0.001$. Eosinophil (identified by flow cytometry as Siglec- $\mathrm{F}^{+} \mathrm{CD} 11 \mathrm{c}^{\mathrm{dim} /} /$ ) frequency and total numbers is shown for lung. ANOVA with Dunnett's multiple comparison test was performed with ${ }^{*} P \leqslant 0.05 ;{ }^{* *} P \leqslant 0.01$. (c) Serial dilutions of digested lung tissue were plated on Sabouraud dextrose agar plates and colony-forming units of individual mice were calculated. Data from three independent experiments were pooled for $\mathbf{c}$.

control mice. However, infected $s t 2^{-1-}$ mice revealed slightly elevated IFN- $\gamma$ synthesis compared with $i l 17 \mathrm{rb} / \mathrm{st} 2^{-1-}$ mice (Supplementary Figure S3). il33 mRNA expression in whole lung tissue was comparable between $s t 2^{-1-}$, ill $17 \mathrm{rb} / \mathrm{st} 2^{-1-}$, and wt mice (data not shown). Although we did not find upregulation of an ILC2-activating cytokine other than IL-33 (e.g., IL-25), we decided to further concentrate on the analysis of the development of IL-13-dependent AAI in IL-25R/IL-33R double-deficient (ill7rb/st $2^{-1-}$ ) mice $^{22}$ to exclude potential redundancy between both cytokines in ILC2 activation if signaling of only one is impaired as we could not exclude residual IL-25 activity. Klein Wolterink et al. ${ }^{28}$ demonstrated that the transcription factor GATA3 is essential for ILC2 development and regulates type 2 cytokine secretion in a quantitative fashion by the copy numbers of GATA3 per cell. We, therefore, analyzed GATA3 expression of ILC2 from wt and $i l 17 \mathrm{rb} / \mathrm{st} 2^{-1-}$ mice by flow cytometry as well as the capability of type 2 cytokine production. The IL-33R did not have a significant influence on GATA3 expression as ILC2 from $i l 17 \mathrm{rb} / \mathrm{st} 2^{-1-}$ mice, and wt mice showed a comparable increase in mean fluorescence intensity of GATA3 with infection (Figure 5a). Subsequently, we next tested whether receptor deficiency would impair ILC2 type 2 cytokine secretion. Therefore, pulmonary leukocytes were isolated from naive and infected wt and ill $17 \mathrm{rb} / \mathrm{st} 2^{-1-}$ mice and restimulated with ionomycin and phorbol 12-myristate acetate for $4 \mathrm{~h}$ in the presence of Brefeldin A. The frequencies of IL-5, IL-13, and IL-5/IL-13 co-producing ILC2 did not differ significantly within the first 2 weeks after infection (data not shown). This changed at 3 weeks post infection with a larger population of IL-5 and IL-13-co-producing cells present in wt mice (Figure 5b) showing that ILC2 from infected $i l 17 r b / s t 2^{-1-}$ 


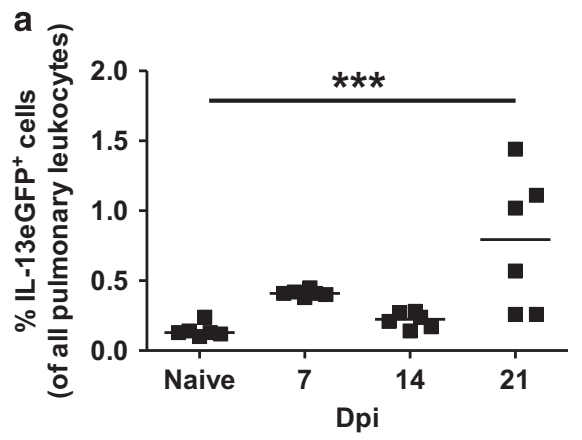

b
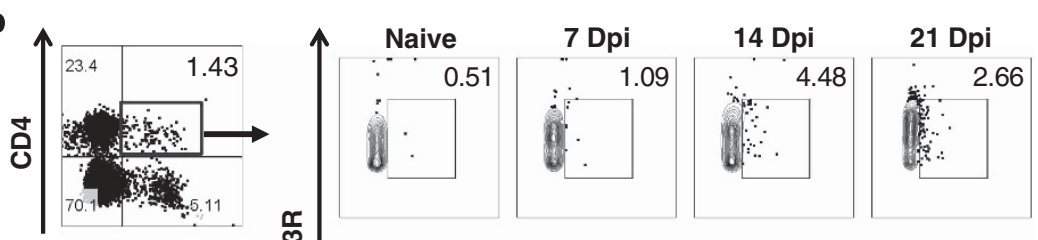

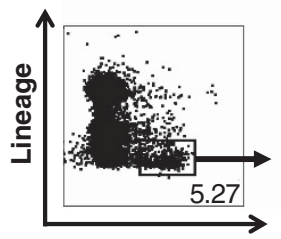

IL-33R

C

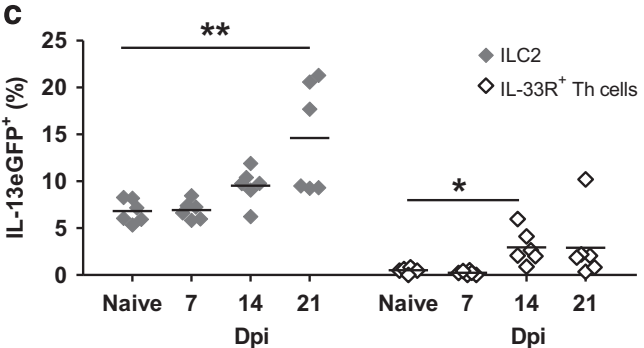

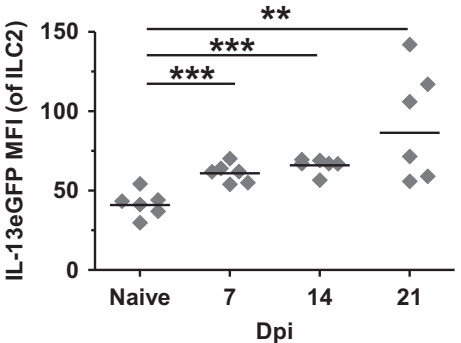

Figure 2 Innate lymphoid cells and T helper (Th) 2 cells contribute to interleukin (IL)-13 production. IL-13-enhanced green flourescence protein (eGFP) reporter mice were infected intranasally with $C$. neoformans 1841 and analyzed at indicated time points ( $n=6$ mice/experiment).

(a) Quantification of IL-13eGFP ${ }^{+}$cells in lung tissue. (b) Pulmonary IL-33R ${ }^{+}$Th cells (left, top, insert) and innate lymphoid cells type 2 (ILC2; left, bottom,

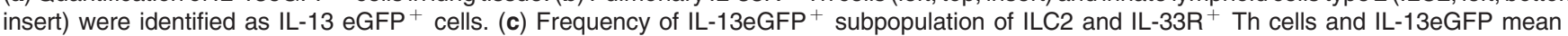
fluorescence intensity (MFI). One-way analysis of variance with Dunnett's multiple comparison test was performed with ${ }^{* \star} P \leqslant 0.01 ;{ }^{* * *} P \leqslant 0.001$. One of two experiments is shown.

mice were impaired to co-produce IL-5 and IL-13 (Figure 5b, $21 \mathrm{Dpi})$. Also, total amounts of IL-5 and IL-13 secreted by pulmonary leukocytes were higher in wt mice $21 \mathrm{Dpi}$ (Figure 5b, c) reflecting higher numbers as well as cytokine secretion capability of ILC2 (Figure 5a, b) and Th2 cells (Figure 5b, c) in wt mice, especially in terms of IL-13. Decreased release of IL-13 was probably responsible for the absent pulmonary mucus production indicated by nonsignificant increase PAS score and gob5 expression in il17rb/ $s t 2^{-1-}$ mice (Supplementary Figure S4) and reduced amounts of both IL-5 and IL-13 translated into almost absent eosinophil recruitment to the lungs of infected ill $7 \mathrm{rb} / \mathrm{st} 2^{-1-}$ mice (Figure 5d). Taken together, these results reveal a non-redundant role predominantly for the IL-33R leading to expansion and type 2 cytokine synthesis by ILC2 during pulmonary cryptococcosis.

\section{Th cell cytokine production and differentiation critically depends on IL-33R signaling}

The IL-33R determines antigen-dependent type 2 cytokine production and polyfunctionality (i.e., coexpression of IL-4, IL-5, and IL-13) in Th cells. ${ }^{17}$ As we have recently shown, regulatory $\mathrm{T}$ cells (Treg) selectively attenuate type 2 responses in cryptococcosis including IL-13 production. ${ }^{29}$ However, Treg subsets have been shown to be dependent on IL-33R signaling themselves for development and proper effector functions, e.g., IL-10 production and immunosuppression. ${ }^{30,31}$ Naive wt and il17rb/st2 $2^{-1-}$ mice harbor comparable Treg numbers, while GATA3-expressing Th cells in lung parenchyma were hardly detectable (Figure 6a, naive plots). This changed after infection as GATA $^{+}$and GATA3 $^{+} /$Foxp3 $^{+}$Th cells could be detected by $21 \mathrm{Dpi}$ in wt mice but not in ill $\mathrm{rb} / \mathrm{st} 2^{-1-}$ mice (Figure 6a) supporting the concept of a positive feedback 

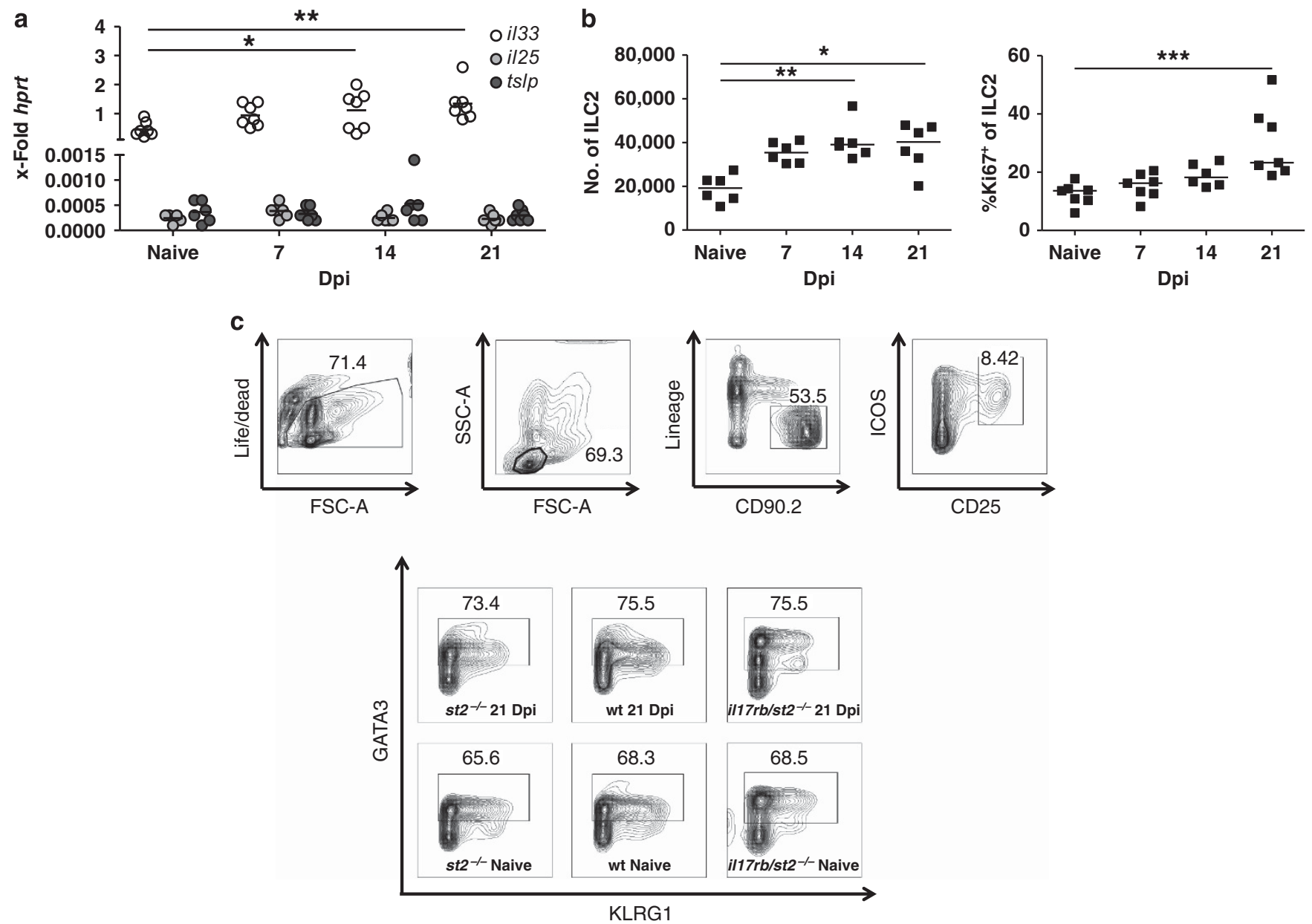

Figure 3 Innate lymphoid cells type 2 (ILC2) proliferation but not GATA3 or KLRG1 expression is mainly driven by interleukin (IL)-33. Wild-type (wt) mice were infected intranasally with $C$. neoformans 1841 and analyzed at indicated time points ( $n=7$ mice/experiment). (a) Expression of $i / 33$, il25, and $t s / p$ was measured in lung tissue by quantitative PCR. (b) Total numbers of pulmonary ILC2 and Ki67 frequency indicative of recent proliferation at indicated time points is shown. (c) Representative flow cytometry plots of gating strategy of pulmonary ILC2 from st2 ${ }^{-1-}$, il17rb/st2 $2^{-1-}$, and wt mice is shown making IL-33R staining dispensable for identification. Kruskal-Wallis test with Dunn's multiple comparison test was performed with ${ }^{\star} P \leqslant 0.05 ;{ }^{\star \star} P \leqslant 0.01 ;{ }^{* \star \star} P \leqslant 0.001$. One of two experiments is shown.

loop between IL-33-mediated GATA3 upregulation and subsequent IL-33R expression. ${ }^{31}$ Indeed, IL-33R ${ }^{+}$Foxp3 $^{-}$and IL-33R ${ }^{+}$Foxp $3^{+}$Th cells from wt mice represented the most prominent GATA3-expressing Th cells after infection (Supplementary Figure S5). Next, we analyzed how the expression pattern of GATA3 and Foxp3 impacts the IL-13 production capability, as we showed earlier that Treg cells have an inhibitory effect on IL-13 production. ${ }^{29}$ We found remarkable IL-13 expression only in GATA3 single-expressing Th cells, while Foxp3 obviously precludes IL-13 production even in GATA3/ Foxp3 double-positive Th cells (Figure 6b). This was also confirmed by the analysis of IL-13 mean fluorescence intensity (data not shown), pointing to a dominant suppressive function of Foxp3 over GATA3 in terms of IL-13 production and thus extending earlier in vitro and in vivo findings. ${ }^{32}$ In conclusion, Th cells are also targeted by IL-33 early after infection and develop into three different subsets based on expression of the transcription factors GATA3 and Foxp3 revealing Treg $\left(\right.$ Foxp $\left.^{+}\right)$, Th2 $\left(\mathrm{GATA}^{+}\right)$, and "Treg/Th2 hybrid cells"
$\left(\mathrm{GATA}^{+} / \mathrm{Foxp}^{+}\right)$. Furthermore, GATA3 single-positive Th cells show a superior IL-13 production capability and are only found with concomitant expression of the IL-33R.

\section{Alternative activation of alveolar macrophages is severely impaired in the combined absence of IL-33R/IL-17RB}

Previously, we and others demonstrated that IL-4R $\alpha$-activated macrophages are unable to provide protection against cryptococcal infection ${ }^{16,33,34}$ and probably promote detrimental dissemination to the brain. ${ }^{16,33}$ These macrophages are called M2 or alternatively activated macrophages. ${ }^{35}$ Furthermore, M2 macrophages are supposed to have a central function in AAI development, ${ }^{36}$ as transfer of IL- $4 \mathrm{R} \alpha^{+/+}$but not IL- $4 \mathrm{R} \alpha^{-1-}$ macrophages into ovalbumin (OVA)-sensitized IL-4R $\alpha^{-1-}$ recipients was sufficient for the development of AAI prior to pulmonary OVA challenge. ${ }^{37} \mathrm{~A}$ M2-like phenotype could also be shown by direct IL-25 and IL-33 stimulation. ${ }^{38,39}$ We isolated alveolar macrophages by flow cytometry-based cell sorting as shown by the representative gate $\left(\right.$ Siglec- $\mathrm{F}^{+}$, 

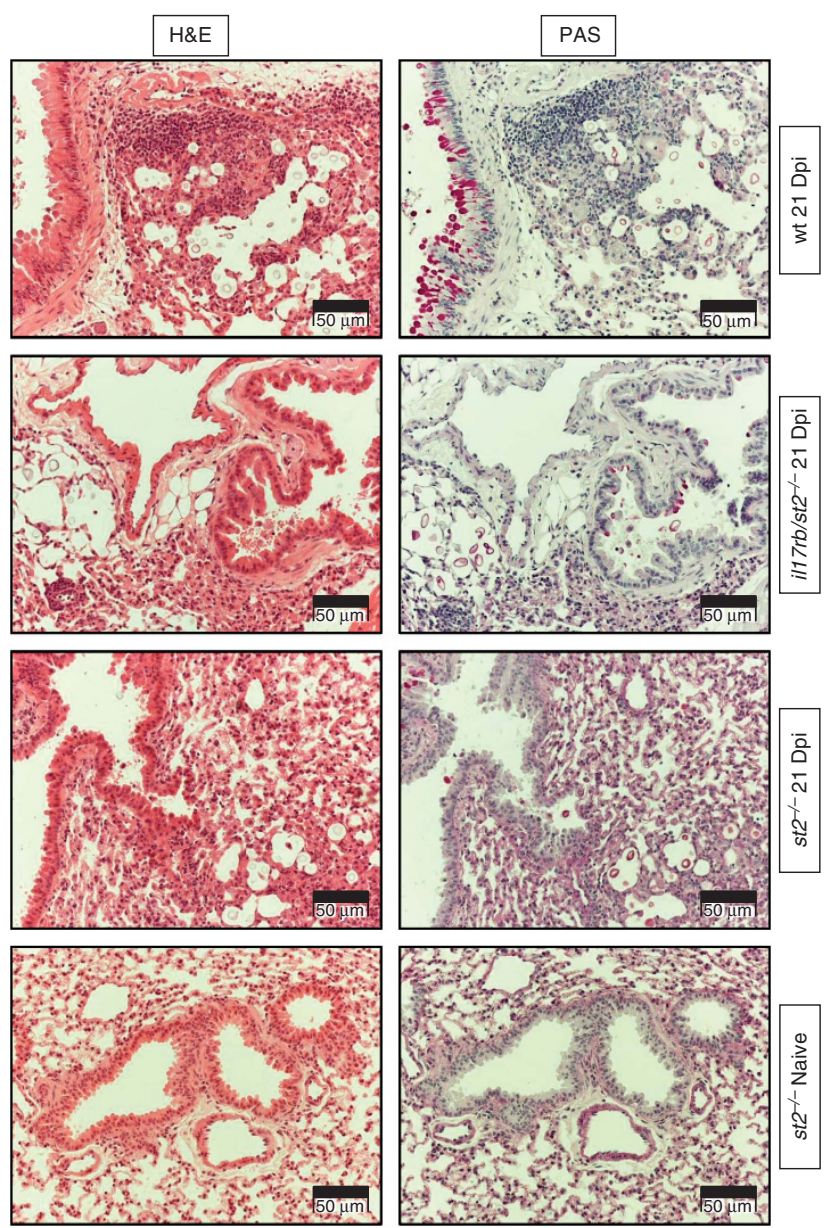

Figure 4 Mucus production is severely reduced in lungs of infected il17rb/st2 ${ }^{-1-}$ and st2 ${ }^{-1-}$ mice. Hematoxylin and Eosin staining (H\&E, left panel) and periodic acid-Schiff (PAS, right panel) staining were performed on representative lung sections from st2 ${ }^{-1-}$, il $17 \mathrm{rb} / \mathrm{st} 2^{-1-}$, and wt mice. Note the comparable fungal loads in $s t 2^{-1-}$, il1 $7 \mathrm{rb} / \mathrm{st}^{-1-}$, and wt mice and prominent PAS staining in the wild-type (wt) sample. Naive st $2^{-1-}$ mouse is shown for comparison.

$\mathrm{CD}_{11 \mathrm{c}^{+}}$) in Figure $7 \mathbf{a}$ and analyzed $\mathrm{M} 2$-associated molecules ${ }^{14,35}$ by quantitative real-time PCR. Indeed, in wt alveolar macrophages we found upregulation of chi3l3, fizz1, and $\operatorname{arg1}$ (Figure $\mathbf{7 b}$ ) that are associated with M2 activation. In contrast, mRNA levels of arg1 and fizz1 in alveolar macrophages from ill $7 \mathrm{rb} / \mathrm{st} 2^{-1-}$ mice hardly increase upon infection, and expression of chi3l3 mRNA is significantly reduced (Figure $\mathbf{7 b}$ ). This was confirmed by fluorescence-based microscopy on sorted alveolar macrophages for detection of Ym1 (protein product of chi3l3) that revealed a significant increase in wt mice that is strongly attenuated in illi rb/st2 $2^{-1-}$ mice (Supplementary Figure S6). Sorted alveolar macrophages of both strains showed comparable and overall negligible inos2 expression (data not shown). Interestingly, although we found markers of alternatively activated wt alveolar macrophages already $14 \mathrm{Dpi}$ (Figure 7b), we did not find remarkable IL-33R expression by flow cytometry before $21 \mathrm{Dpi}$ (data not shown) or quantitative PCR analysis (Figure 7b). In summary, the type 2 effector status including alternative activation of macrophages found during the first 3 weeks upon pulmonary infection with C. neoformans strain 1841 selectively leads to AAI without affecting fungal control during this early time period (data not shown and Figure 1c).

\section{ILC2 fail to initiate type 2 responses in the absence of adaptive immunity in pulmonary cryptococcosis}

As we identified two potential IL-13 sources, we asked if they would require each other for the induction of type 2 immunopathology in cryptococcosis. To answer this question, we compared infected rag2 ${ }^{-1-}$ mice that cannot develop mature T cells and B cells but are able to give rise to ILC2 and $\mathrm{rag} 2 / \gamma \mathrm{c}^{-1-}$ mice, which additionally lack ILC. ${ }^{40,41}$ ILC2 from infected rag2 $2^{-1-}$ mice did not show enhanced proliferation within 14 Dpi compared with naive rag2 $2^{-1-}$ mice (data not shown). However, ILC2 from naive rag2 $2^{-1-}$ mice showed increased basal Ki67 staining compared with naive wt ILC2 (Figures 3b, 8a, b; $P=0.046$ two-tailed $t$-test). This could be a consequence of reduced proliferation control under homeostatic conditions in rag2 ${ }^{-1-}$ mice as the total numbers of ILC2 in naive rag2 ${ }^{-1-}$ mice were comparable to those from infected wt mice $21 \mathrm{Dpi}$ that were a result of ILC2 expansion (Figures 3b, 8b). Although the numbers of ILC2 found in rag $2^{-1-}$ mice should be sufficient to induce type 2 immunity, we neither found enhanced expression of markers indicating alternative (alveolar) macrophage polarization, enhanced eosinophil recruitment, or mucus production in the airways of infected animals (data not shown), nor could we find enhanced induction of type 2 cytokines IL-5 and IL-13 (Supplementary Figure S7). Even 50 Dpi no IL-13 was found in supernatants of stimulated pulmonary leukocytes from rag1 ${ }^{-1-}$ mice (M. Eschke et al., unpublished). Conclusively, ILC2 were unable to induce type 2-associated pathology in the absence of adaptive immunity.

\section{DISCUSSION}

IL-13, signaling via the type 2 IL-4R, was initially identified as a key cytokine ${ }^{42,43}$ in the context of AAI development in an experimental sensitization model using OVA. ${ }^{44}$ We reported IL-13 contribution to immunopathology in chronic pulmonary cryptococcosis as IL-13 $3^{-1-}$ mice were largely resistant against intranasal infection with strain $1841 .{ }^{14,16}$ Th2 cells were identified as the dominant population of IL-13 synthesis late in infection. ${ }^{11,17}$ However, the early development of the antifungal type 2 immune response remained elusive so far. In the current study, we identified two IL-13-competent populations of cells very early after infection, one being ILC2 and the other Th2 cells. Our data revealed that IL-13 secretion is associated with expression of IL-33 in pulmonary cryptococcosis. The source(s) of IL-33 and the mechanism(s) of release are under current investigation but non-hematopoietic cells emerged to be a potential source (L. Heyen et al., unpublished). Only recently, an elegant study highlighted the role of processed chitin polymers in the instruction of dendritic cells to initiate Th2 differentiation of naive Th cells in pulmonary 

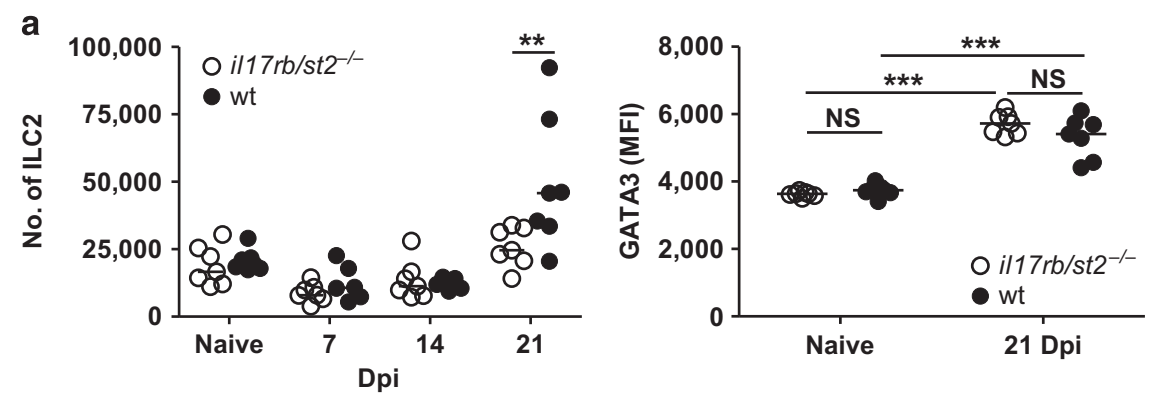

b
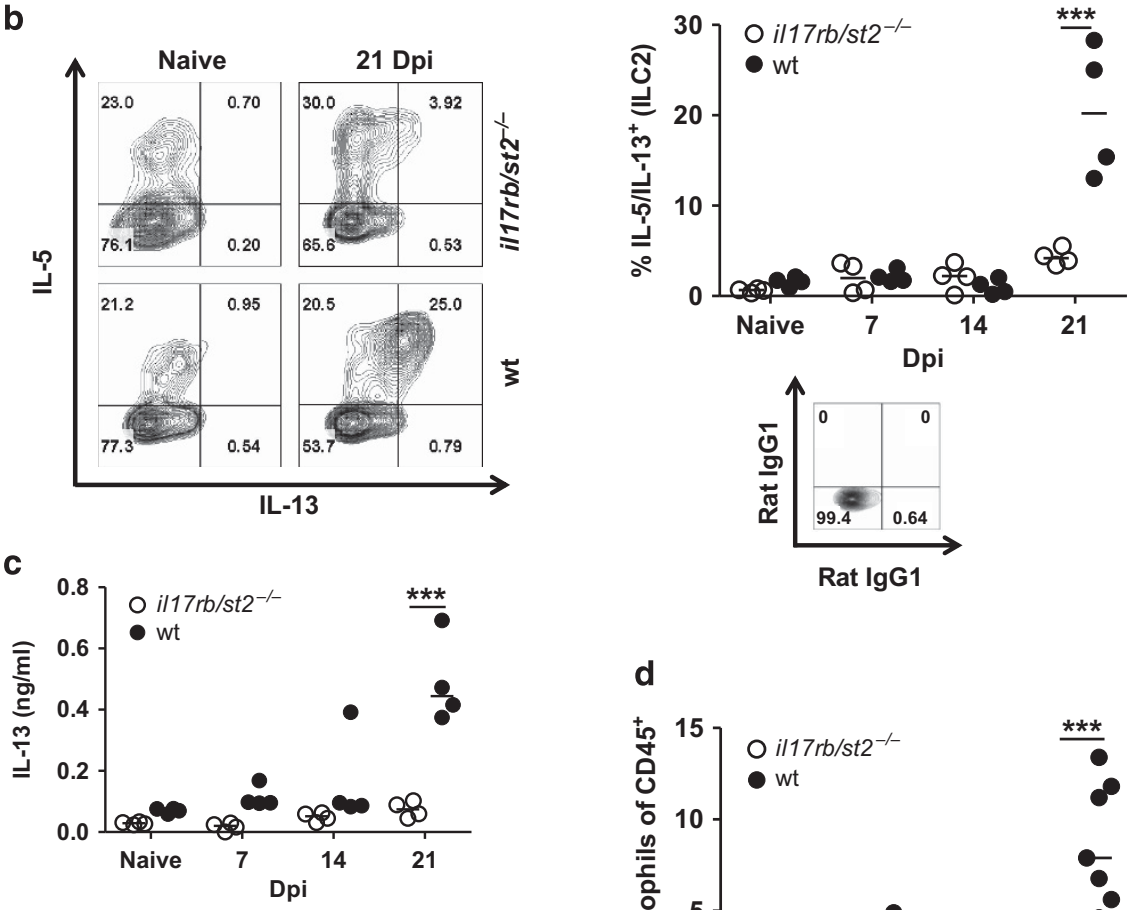

Rat IgG1
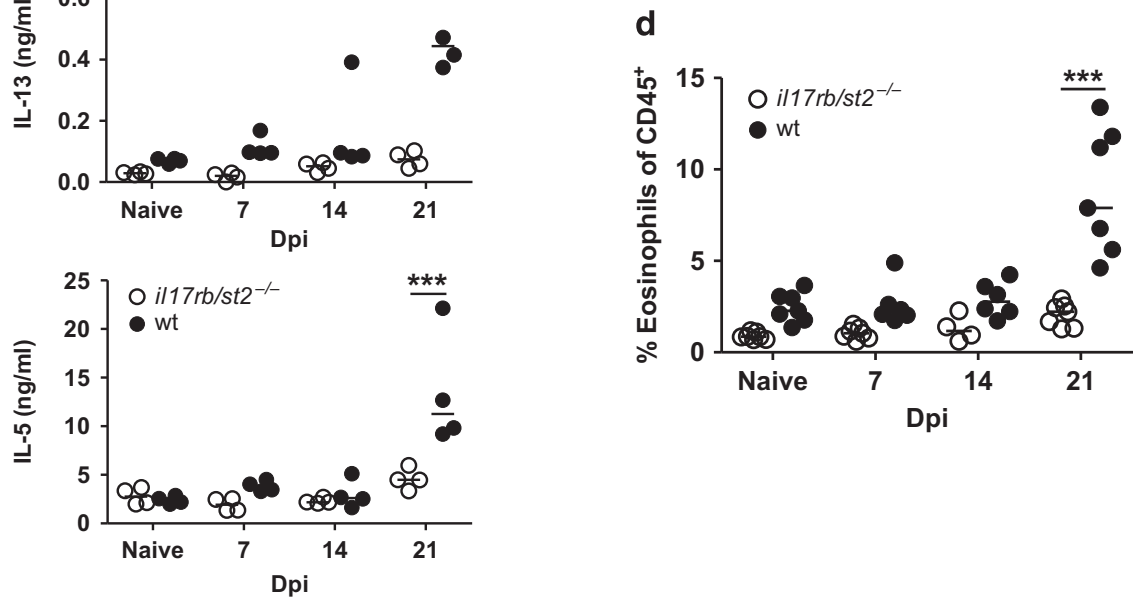

Figure 5 Innate lymphoid cells type 2 (ILC2)-derived interleukin (IL)-13 and type 2 immunopathology is reduced in il17rb/st2 ${ }^{-1-}$ mice. Wild-type (wt) and il17rb/st2 $2^{-1-}$ mice were infected intranasally with $C$. neoformans 1841 and analyzed at indicated time points $(n=7$ mice/experiment). (a) Total numbers, GATA3 frequency and mean fluorescence intensity (MFI) of pulmonary ILC2 are shown. (b) Pulmonary leukocytes from individual mice were restimulated with phorbol 12-myristate 13-acetate and ionomycin for $4 \mathrm{~h}$ in the presence of Brefeldin A and intracellular staining of IL-5 and IL-13 in ILC2 was performed. Frequency analysis of IL-5/IL-13 double-positive ILC2 at indicated time points ( $n=4$, remaining three mice per group were used for cell sorting). (c) Supernatant analysis from mice shown in (b) with ELISA for IL-5 and IL-13 (individual mice, w/o Brefeldin A). (d) Frequency of pulmonary eosinophilic granulocytes indicative for type 2 inflammation of pulmonary leukocytes at indicated time points. For all data shown one-way analysis of variance with Bonferroni's multiple comparison test was performed with ${ }^{\star} P \leqslant 0.05$; ${ }^{\star *} P \leqslant 0.01 ;{ }^{\star \star \star} P \leqslant 0.001$. One of two experiments is shown.

cryptococcosis. ${ }^{45}$ It remains to be determined whether other cells may also be affected by chitin fragments and if chitin can contribute to IL-33 liberation.

Previously, we showed IL-33 to critically regulate the amounts of type 2 cytokines in an antigen-dependent manner by the development of polyfunctional Th2 cells characterized by simultaneous production of IL-4, IL-5, and IL-13. ${ }^{17}$ These signature type 2 cytokines are classically linked to the expression of the Th2 cell key transcription factor GATA3. ${ }^{28,46}$ Here, we showed that $i l 17 \mathrm{rb} / \mathrm{st} 2^{-1-}$ mice are almost unable to give rise to GATA3-expressing Th cells. Interestingly, it was previously demonstrated in certain tissues, such as the dermis, a remarkable proportion of Treg cells not only expresses Foxp3 but also GATA3. ${ }^{32}$ Under homeostatic conditions, pulmonary 


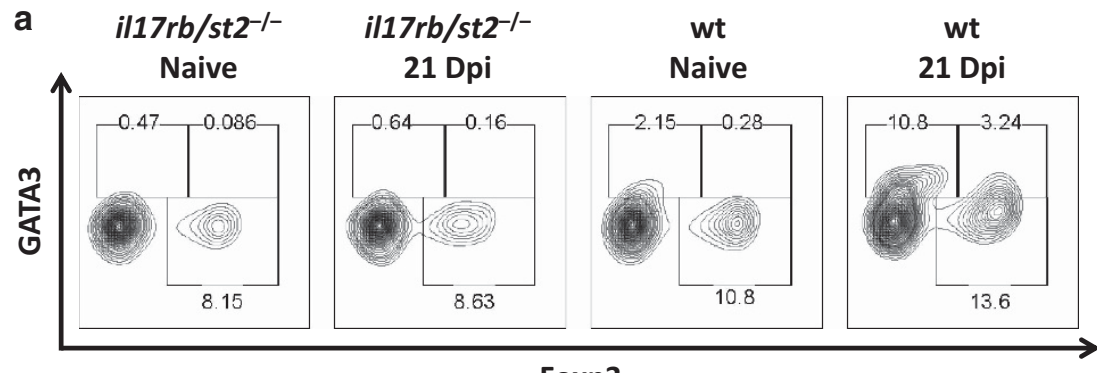

Foxp3
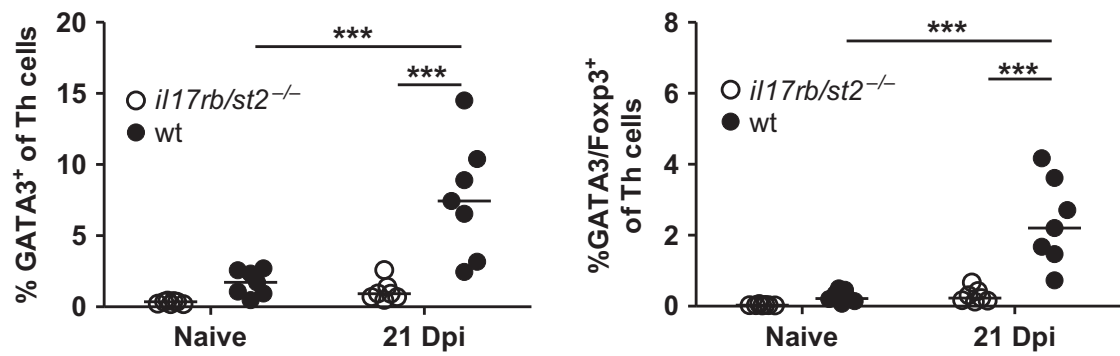

b

wt wt
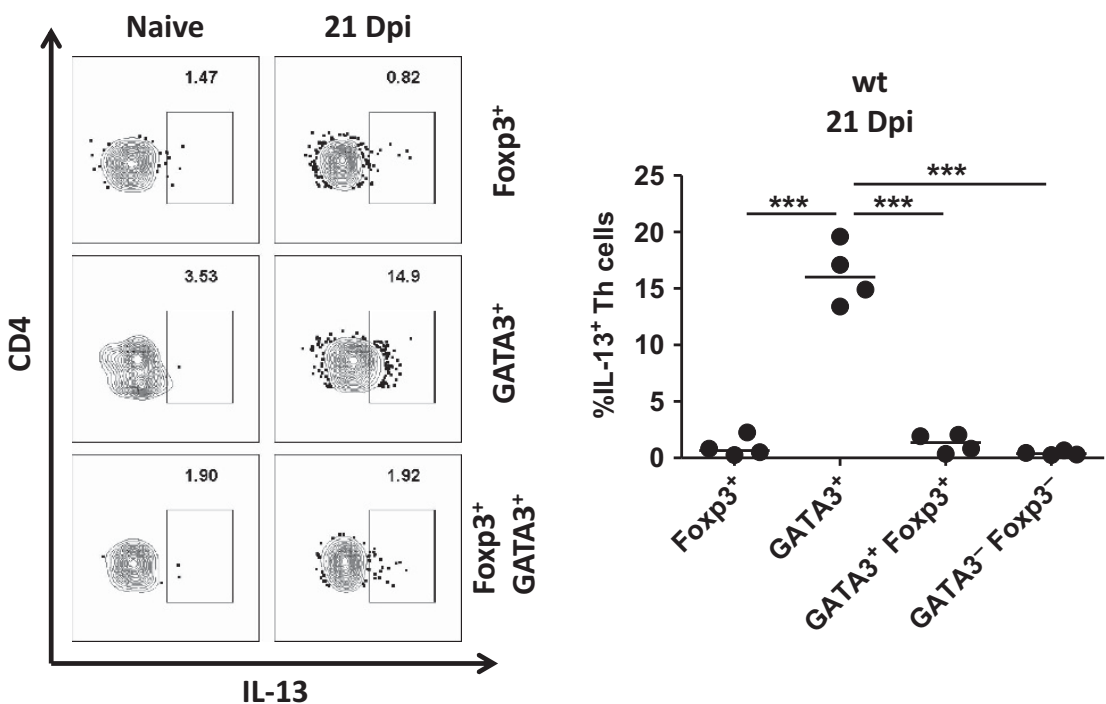

Figure 6 Infection induces IL-33R-dependent upregulation of GATA3 and Foxp3 in Th cells. Wild-type (wt) and il17rb/st2 ${ }^{-1-}$ mice were infected intranasally with $C$. neoformans 1841 and analyzed at indicated time points ( $n=7$ mice/experiment). (a) Representative FACS plots of pulmonary Th cells analyzed for expression of Foxp3 and GATA3 by intracellular staining for naive and 21 days post infection (Dpi) mice. Frequency of GATA3 ${ }^{+}$and GATA3/ Foxp3 ${ }^{+}$pulmonary Th cells is shown. (b) Pulmonary leukocytes from individual mice were restimulated with PMA/iono for $4 \mathrm{~h}$ in the presence of Brefeldin A and intracellular staining of IL-13 was performed. Representative FACS plots are shown for frequencies of IL-13 ${ }^{+}$Th cell subpopulation for naive and $21 \mathrm{Dpi}$ wt mice as GATA3 ${ }^{+}$subpopulations are almost undetectable in il17rb/st2 ${ }^{-1}$ mice. For presented data analysis of variance with Bonferroni's multiple comparison test was performed with ${ }^{\star} P \leqslant 0.05 ;{ }^{\star \star} P \leqslant 0.01 ;{ }^{* \star} P \leqslant 0.001$. One of two experiments is shown.

Treg cells in our hands showed only modest levels of GATA3 (Figure 6a). Interestingly, not only GATA3 ${ }^{+}$cells developed during the first 21 Dpi but also GATA3/Foxp3 co-expressing Th2/Treg hybrid cells that were also absent in il17rb/st2 ${ }^{-1-}$ mice pointing to a common way of differentiation. Here, we show the dependency of GATA3/Foxp3 co-expressing Th cells on the IL-33R in a pulmonary fungal infection model for the first time. As we demonstrated earlier, Treg cells display a role in fungal growth control early after the infection and selectively suppress Th2 immune responses as the absence of
Treg cells induced by diphtheria toxin application in DEREG mice led to exacerbation of Th2 immune responses. ${ }^{29}$ Therefore, we were surprised to find IL-33R-dependent Foxp3/ GATA3-expressing cells and Foxp3 may thus not only suppress IL-13 in these Th2/Treg hybrid cells but also drive them to suppress IL-13-producing GATA3 single-positive Th2 cells. IL-33 has been previously shown to induce its own receptor and GATA3 in resting Th2 cells with subsequent IL-13 production. ${ }^{47}$ In terms of IL-13 secretion Th2/Treg hybrid cells were of lower importance in pulmonary cryptococcosis and may 

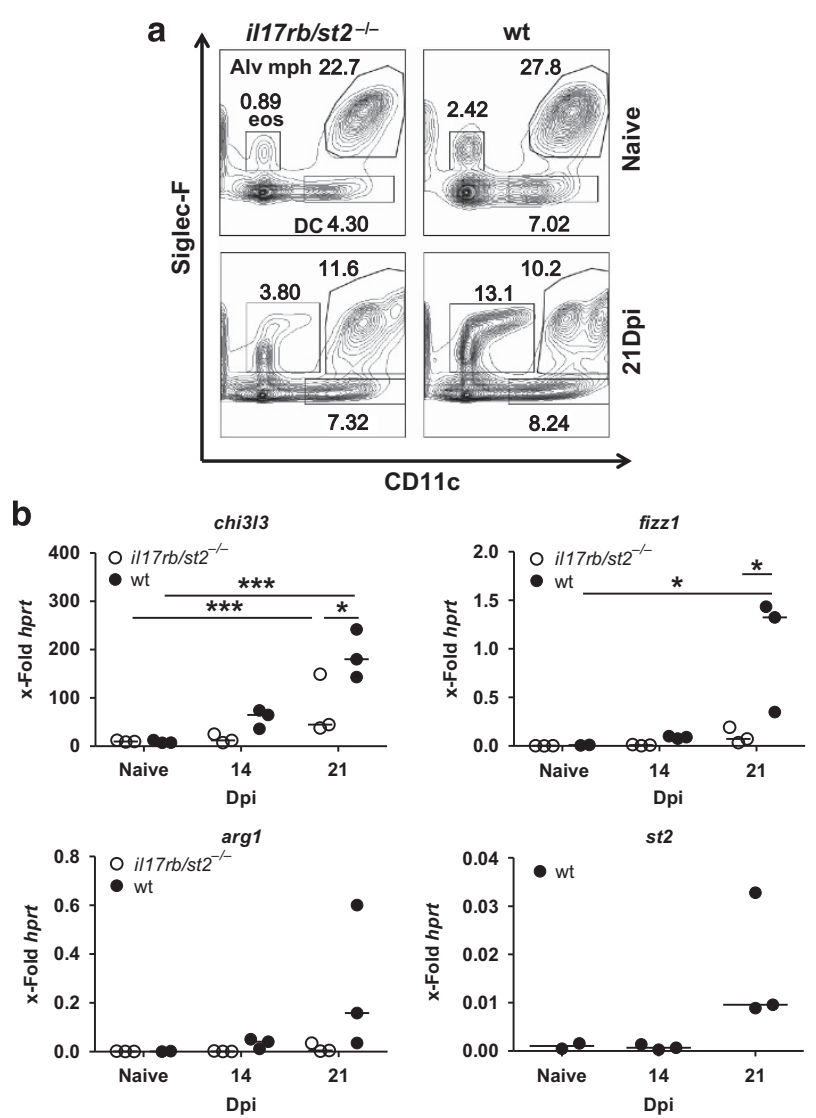

Figure 7 Alternative activation of alveolar macrophages is severely attenuated in the absence of interleukin (IL-13) and IL-33 signaling. Wild-type (wt) and $i l 17 \mathrm{rb} / \mathrm{st}^{-1}-$ mice were infected intranasally with C. neoformans 1841 and analyzed at indicated time points ( $n=3$ mice/ experiment). (a) Gating scheme for isolation of alveolar macrophages (alv mph, Siglec-F ${ }^{+} / \mathrm{CD}_{11 \mathrm{c}^{+}}$) is given for discrimination from eosinophilic

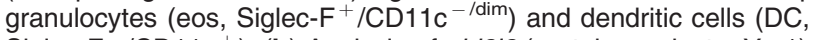
Siglec- ${ }^{-} / \mathrm{CD}_{11 c^{+}}$). (b) Analysis of $c h i 3 / 3$ (protein product $=\mathrm{Ym} 1$ ) and st2 (wt) expression by quantitative real-time PCR in sorted alveolar macrophages at indicated time points. Analysis of variance with Bonferroni's multiple comparison test was performed with ${ }^{\star} P \leqslant 0.05$; ${ }^{\star \star \star} P \leqslant 0.001$. One of two experiments is shown.

represent a transitional stage of Treg cells. Theoretically, IL-33R could have a comparable role in "Th2 programming" of Treg cells as shown for the IL- $4 \mathrm{R}^{48}$ thereby limiting Treg suppression capability with respect to (IL-33R ${ }^{+}$GATA $^{+}$) Th2 effector cells. On the other hand, in the intestinal tract it was demonstrated that Treg differentiation, recruitment, and function is dependent on IL-33 $\mathrm{R}^{31}$ as well as GATA3 (ref. 32) expression. Already immune homeostasis depends on GATA3 expression in Treg cells with evidence of direct regulation of Foxp3 by GATA3 as the conserved non-coding DNA sequence 2 of the foxp 3 locus binds GATA $3 .{ }^{49}$ Of note, in asthmatic children an impaired function of Treg cells in terms of type 2 immune response suppression has been proposed. ${ }^{50}$ Furthermore, this loss of suppressive control may be even Th cell subset-specific as recently shown in a mouse model. ${ }^{51}$ Therefore, the functional properties of these Foxp3/GATA3 hybrid cells in terms of Cryptococcus-induced AAI undoubtedly
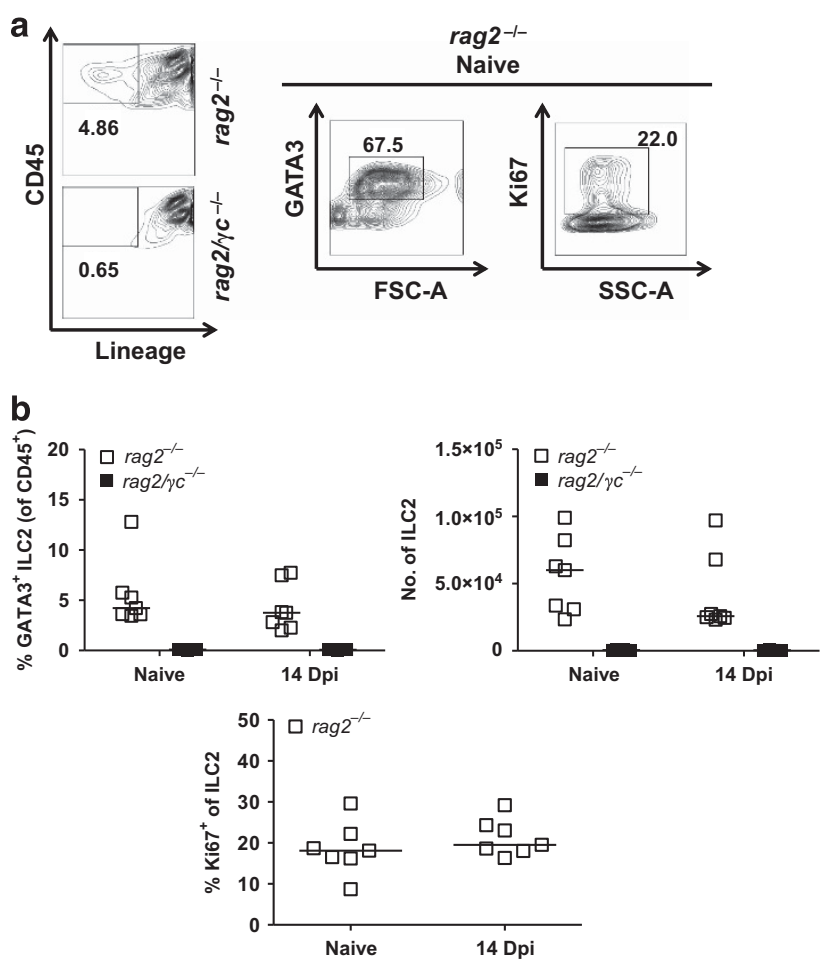

Figure 8 Innate lymphoid cells type 2 (ILC2) are unable to induce type 2 inflammation in pulmonary cryptococcosis without adaptive immunity. rag $2^{-1-}$ and rag2 $/ \gamma \mathrm{C}^{-1-}$ mice were infected intranasally with $C$. neoformans 1841 and analyzed at indicated time points ( $n=7$ mice/experiment). (a) Gating scheme for ILC2 revealed ILC2 in rag2 ${ }^{-1-}$ (left, top) but not rag2/ $\mathrm{C}^{-1-}$ (left, middle) mice. rag2 ${ }^{-1-}$ ILC2 express GATA3 (middle) and Ki67 indicates recent proliferation (right). (b) Frequency and total numbers of pulmonary leukocytes. Ki67 frequency reveals no increase of proliferation after infection. One of two experiments is shown.

require further investigation and may be valuable for therapeutic approaches.

The use of $i l 17 \mathrm{rb} / \mathrm{st} 2^{-1-}$ mice also allowed to investigate a system where ILC2 are present but cannot be properly activated by IL- 25 and IL- $33 .{ }^{52}$ Our data revealed a superior role for IL-33R over IL-25R as the expression of the latter is almost undetectable in naive as well as infected mice. Of note, we were not able to detect IL-17RB on pulmonary cells by flow cytometry in naive and infected wt mice (data not shown). Yet, we cannot completely rule out a role for IL-25 in the pulmonary immune response against $C$. neoformans, as we did not analyze il17rb $\mathrm{rb}^{-1-}$ mice. Neill et al. ${ }^{22}$ showed in the N. brasiliensis model that rag2 ${ }^{-1-}$ mice develop type 2 inflammation even when ILC2 are the sole source of IL-13 but parasite clearance in the absence of Th cells was not achieved. Interestingly, parasite expulsion was successful after reconstitution with IL-13 ${ }^{-1-}$ Th cells, indicating that Th cells are necessary but not their production of IL-13. We showed for detrimental antifungal type 2 immunity that IL-13-competent ILC2s were unable to induce AAI without the support of Th2 cells in rag2 $2^{-1-}$ mice. This may result from lower quantities of IL-33 that are released in our model in contrast to models using excessive administration of exogenous IL-33, ${ }^{53}$ enzymatic 
activity-containing agents, ${ }^{54}$ or infections ${ }^{22,55}$ with heavy tissue destruction and inflammation resulting in strong activation of ILC2, whereas low-dose infection or models using antigens with sensitizing character may rely on Th cells to induce type 2 responses. ${ }^{56}$ In a model of combined allergen exposure, rag $1^{-1-}$ mice were also not capable of producing IL-13 or recruiting eosinophils in numbers comparable to wt counterparts. ${ }^{24}$ Th cells may provide growth factors, e.g., IL-2, that are necessary for proliferation and proper activation of ILC2 (refs. 56, 57) when no excessive tissue destruction is present. Of note, wt Th cells from $21 \mathrm{Dpi}$ mice show enhanced il2 expression compared with Th cells from illitrb/st $2^{-1-}$ mice (Supplementary Figure S8). The dissection of this cooperative network could be of clinical relevance for the development of new therapeutic approaches against asthma phenotypes that may be associated with ILC2, as it is known that ILC2 cytokine production is hardly influenced by conventional steroid administration. ${ }^{2,58}$

Macrophages are central in the defense against C. neoformans and activation by type 2 cytokines allows intracellular and extracellular fungal proliferation. ${ }^{33,34,59,60}$ In this study, wt mice produced significantly more IL-13 in comparison with il17rb/ $s t 2^{-1-}$ mice (Figure 5c) and only wt alveolar macrophages showed significantly increased alternative activation (Figure 7b). Concomitant with enhanced IL-33R expression in alveolar macrophages (Figure $7 \mathbf{b}$ ) that may amplify alternative polarization, this scenario would provide an ideal refugium for cryptococcal proliferation that may be the reason for higher fungal loads observed later in infection. ${ }^{17}$ Together, this could argue for a model of synergism between IL-13 and IL-33 in alternative macrophage activation as demonstrated in vitro by Kurowska-Stolarska. ${ }^{61}$ Thus, early IL-13 may be responsible for the observed alternative activation that is time-dependently strengthened by IL-33R upregulation on alveolar macrophages leading to superior alternative activation in wt mice. IL-33 appears to contribute by amplification rather than induction of alternative macrophage activation as in C. neoformans-infected IL-4 and IL-13 single-deficient and IL-4R $\alpha$ knockout mice, almost no alternative activation can be detected although IL-33 signaling is intact. ${ }^{14}$ Consistent with that, alternative macrophage activation on a low level is detectable in $i l 17 \mathrm{rb} / \mathrm{st} 2^{-1-}$ mice as shown in Figure $\mathbf{7 b}$. Interestingly, a recently published report on malaria provided evidence for a role of alternatively activated macrophages in promoting the development of regulatory $\mathrm{T}$ cells ${ }^{62}$ thus providing an additional explanation (besides a direct response to IL-33) for the lower frequencies of Foxp3 single-positive and Foxp3/GATA3 double-positive Th cells seen in the lungs of C. neoformans-infected il17rb/ $s t 2^{-1-}$ mice.

In summary, we present a model of pulmonary mycosis with a ubiquitous fungal pathogen that results in the development of AAI in an IL-33R-dependent manner. ILC2 and GATA3 ${ }^{+}$Th2 cells contribute to IL-13 production and an IL-33R-dependent hybrid GATA3 ${ }^{+} /$Foxp $^{+}$Treg/Th2 subpopulation develops that does not contribute to IL-13 production despite GATA3 expression. Alternative macrophage activation indicating a type 2 immune response was readily found after infection and may be used in a clinical setting as a type 2 surrogate, as they are obtainable by bronchoalveolar lavage. This argues for a regulatory role of IL-33 in the development of AAI that is independent of excessive tissue destruction and sole antigen load in a model of live fungal infection making this model highly suitable for future research in fungus-induced AAI.

\section{METHODS}

Mice. Eight to twelve-week-old female wt (Janvier, Le Genest Saint Isle, France), IL-13eGFP reporter, and $i l 17 \mathrm{rb} / \mathrm{st} 2^{-/-}$(kindly provided by Dr A.N.J. McKenzie, LMB Cambridge, UK), st2 ${ }^{-}$mice (kindly provided by Dr T. Kamradt, Friedrich-Schiller-University, Jena, Germany), $r a g 2^{-1-}$ and $r a g 2 / \gamma c^{-1-}$ (kindly provided by Dr A. Diefenbach, Albert-Ludwigs-University, Freiburg, Germany) mice on BALB/c background were maintained in an individually ventilated cages system under specific pathogen-free conditions and in accordance with the guidelines approved by the Animal Care and Usage Committee of the "Landesdirektion Leipzig" (accreditation no. 24-9168.11/18/36). Sterile food and water were provided ad libitum. Mice were tested regularly for pathogens according to the recommendations for health monitoring of mice provided by the Federation of European Laboratory Animal Science Associations accreditation board. Infected mice were monitored daily for survival and morbidity.

C. neoformans and infection of mice. C. neoformans strain 1841 (serotype D) was kept at $-80{ }^{\circ} \mathrm{C}$ in $10 \%$ skimmed milk and expanded in Sabouraud dextrose medium ( $2 \%$ glucose, $1 \%$ peptone; SigmaAldrich, Munich, Germany) overnight on a shaker at $30^{\circ} \mathrm{C}$. After cultivation, fungal cells were washed twice with sterile phosphatebuffered saline (PBS), and counted in a hemacytometer. Inocula were finally diluted in PBS $(2.5 \times \mathrm{E} 4 / \mathrm{ml})$ for intranasal infection. Mice received $20 \mu \mathrm{l}$ containing 500 colony-forming units. Prior to infection, mice were anesthetized by intraperitoneal application of a 1:1 mixture of ketamine (100 mg/ml; Ceva Tiergesundheit, Düsseldorf, Germany) and xylazine $(20 \mathrm{mg} / \mathrm{ml}$; Ceva Tiergesundheit).

Preparation and stimulation of pulmonary leukocytes, determination of fungal lung burden. For preparation of lung leukocytes, lungs were perfused with sterile $0.9 \%$ sodium chloride solution. After removal, lungs were cut into small pieces and digested for $30 \mathrm{~min}$ at $37^{\circ} \mathrm{C}$ in RPMI1640 (with $10 \%$ heat-inactivated fetal bovine serum (FBS) and $1 \%$ penicillin/streptomycin) supplemented with Collagenase $(0.689 \mathrm{mg} / \mathrm{ml}$; Roche Diagnostics Deutschland $\mathrm{GmbH}$, Mannheim, Germany), $10 \mu \mathrm{M}$ sodium pyruvate, and DNase IV (111 U/ $\mathrm{ml}$; Sigma-Aldrich). Following passage through a $100-\mu \mathrm{m}$ nylon mesh (BD Biosciences, Heidelberg, Germany), the filtrate was resuspended in $1 \mathrm{ml}$ PBS containing 3\% FBS (Invitrogen, Darmstadt, Germany) and $50 \mu \mathrm{l}$ were used for colony-forming unit enumeration. Therefore, serial dilutions were plated on Sabouraud dextrose agar plates and colonies were counted after an incubation period of $48 \mathrm{~h}$ at $30^{\circ} \mathrm{C}$. Remaining cells were treated with Gey's solution (1 min on ice) to lyse erythrocytes before checking viability and calculating total cell number using a Neubauer improved counting chamber (Laboroptik, Lancing, UK). Afterwards, cells were washed with PBS containing 3\% heat-inactivated FBS and samples were taken for ex vivo phenotyping of cells. Some mice of a group $(n=3)$ were subsequently used for cell sorting. Pulmonary leukocytes of remaining mice of the same group $(n=4)$ were resuspended in 70\% Percoll (GH Healthcare Biosciences AB, Uppsala, Sweden) and layered under 30\% Percoll. After centrifugation leukocytes were recovered from interphase, washed with Iscove's Modified Dulbecco's Medium (IMDM) (PAA Laboratories, Cölbe, Germany) and used for in vitro restimulation and subsequent cytokine staining. For ex vivo phenotyping $5 \times \mathrm{E} 5$ cells were used and for intracellular cytokine staining at least $1 \times \mathrm{E} 6$ cells were acquired. Purified cells were adjusted to $5 \times \mathrm{E} 6 / \mathrm{ml}$ in IMDM (with $10 \% \mathrm{FBS}$ and 
$1 \%$ penicillin/streptomycin) and stimulated fo $4 \mathrm{~h}$ with $1 \mu \mathrm{g} / \mathrm{ml}$ ionomycin (iono, Sigma Aldrich, Schnelldorf, Germany) and 40ng/ml phorbol 12-myristate 13-acetate (Enzo Life Sciences, Lörrach, Germany) or left unstimulated. For blockade of cytokine secretion and subsequent intracellular cytokine staining Brefeldin A $(5 \mu \mathrm{g} / \mathrm{ml}$; Sigma-Aldrich) was added.

Quantitative real-time PCR. Tissue from lung (middle right lobe) was snap-frozen in liquid nitrogen and stored at $-80^{\circ} \mathrm{C}$ until RNA isolation. Tissue was homogenized by use of peqGOLD TriFast (Peqlab, Erlangen, Germany) and the Precellys Ceramic Kit $(1.4 \mathrm{~mm}$; Peqlab, Erlangen, Germany) at 5,000 r.p.m. for $15 \mathrm{~s}$ in a 24 Precellys homogenisator (Peqlab). FACS-sorted cells were frozen and stored in peqGOLD TriFast. Following extraction using chloroform, RNA was precipitated with isopropanol, washed two times with $70 \%$ ethanol (all: Roth, Karlsruhe, Germany) and resuspended in DEPC water (Roth, Karlsruhe, Germany). In the presence of RiboLock RNase Inhibitor (20 units per sample; Thermo Fisher Scientific GmbH, Schwerte, Germany) RNA was treated with RNase-free DNase I (2.5 units per sample; Thermo Fisher Scientific GmbH, Schwerte, Germany) to eliminate genomic DNA. Afterwards RNA was transcribed to complementary DNA with High capacity cDNA Reverse Transcription Kit (Applied Biosystems, Darmstadt, Germany) using a blend of random/oligo(dT)18 primer according to manufacturer's instruction. Quantitative PCR was performed in duplicates with the iTaq TM Universal SYBR Green Supermix (Bio-Rad, München, Germany) in an iCycler iQ5 (Bio-Rad). Following primers were used for quantitative PCR: il5_forward (for): $5^{\prime}$-TGACAAGCAATGAGA CGATGA-3' and il5_reverse (rev): 5'-CGCCACACTTCTCTTTTT GG-3' (GenBank Accession No. NM 010558; position 171-191 and 359-340). gob5_for: 5'-AGCGTCACAGCCTTGATT-3' and gob5_ rev: 5'-GGACGTGGTGGGTTCATTCT-3' (GenBank Accession No. NM_017474; position 1,907-1,924 and 2,175-2,156), il13_for: $5^{\prime}-\mathrm{AC}$ AAGACCAGACTCCCCTGT-3' and il13_rev: 5'-GGCTGGAGACC GTAGTGGGG-3' (GenBank Accession No. NM_008355; position 200-219 and 378-359), il25_for: 5'-AGCAGGGCCATCTCTCCT T-3' and il25_rev: 5' -AGCCAAGGAGACTCGGTAGA-3' (GenBank Accession No. NM_080729; position 302-320 and 553-534), il33_for: 5'-GGAAAGAACCCACGAAAAGA- ${ }^{\prime}$ and il33_rev: 5'-TGTACTC AGGGAGGCAGGAG-3' (GenBank Accession No. NM_001164724; position 268-287 and 455-436), st2_for: 5'-GCGGAGAATGGAAC CAACTA- $3^{\prime}$ and st2_rev: 5'-AAGCAAGCTGAACAGGCAAT-3 ${ }^{\prime}$ (GenBank Accession No. NM_001164724; position 268-287 and 455-436), inos2_for: 5'-GTGGTGACAAGCACATTTGG-3' and inos2_rev: 5'-GGACAGCTTCTGGTCGATGT-3' (GenBank Accession No. NM_010927; position 1,927-1,946 and 2,103-2,084), fizz1_for: 5'-AGGAACTTCTTGCCAATCCA-3' and fizz1_rev: 5'-CGAGTAAGCACAGGCAGTTG-3' (GenBank Accession No. NM_020509; position 209-228 and 400-381), tslp_for: 5'-AAGTTCG AGCAAATCGAGGA-3' and tslp_rev: 5'-GTGCCATTTCCTGAGT ACCG-3' (GenBank Accession No. NM_021367; position 165-184 and 360-341), chi313_for: $5^{\prime}$-TTGCGTGACTATGAAGCATTG-3' and chi313_rev: 5'-TGAATATCTGACGGTTCTGAGG-3' (GenBank Accession No. NM_009892; position 263-283 and 401-380). The results are illustrated as relative expression compared with expression of hypoxanthine-guanine phosphoribosyltransferase, which was chosen as stable reference gene. Hypoxanthine-guanine phosphoribosyltransferase expression was determined using hypoxanthineguanine phosphoribosyltransferase_for: $5^{\prime}$-GTTGGATACAGGCCA GACTTTGTTG- $3^{\prime}$ and hypoxanthine-guanine phosphoribosyltransferase_rev: 5'-GATTCAACTTGCGCTCATCTTAGGC-3' (GenBank Accession No. NM_013556; positions 660-684 and 822-798) as primers.

Histopathology. Lung samples from naive and infected mice were processed for histological analysis as described previously. ${ }^{17}$ The percentage of PAS-positive goblet cells in bronchial tissue was determined by an independent investigator by counting PAS-positive and PAS-negative bronchial epithelial cells of 5 cross sections of proximal bronchi of 2 slices per lung (different lung regions) with a total of 10 cross sections per mouse. This is modified from Fulkerson et al. ${ }^{63}$ and termed PAS score in this manuscript.

Immunofluorescence microscopy staining of sorted alveolar macrophages and detection of iNOS and Ym1 expression. $2 \times 10^{4}$ sorted alveolar macrophages per sample were cytospun on a poly-LLysine coated slide using a Shandon Cytospin 4 centrifuge (Thermo Scientific) at 1,000 r.p.m. for $10 \mathrm{~min}$ with a two funnel system for two spots per slide, incubated in tap water for $10 \mathrm{~s}$ and air dried. The samples were stored at $-80^{\circ} \mathrm{C}$ until use. For detection of iNOS and Ym1 by immunofluorescence, the slide was fixed with $1 \%$ paraformaldehyde for $10 \mathrm{~min}$, washed, and treated for $2 \mathrm{~min}$ in citrate buffer, $\mathrm{pH} 6$, in a microwave oven $\left(95^{\circ} \mathrm{C}, 600 \mathrm{~W}\right)$. After blocking in $10 \%$ normal donkey serum (United States Biological, Salem, MA) in PBS with $0.1 \%$ Triton X-100 (PBSTx, AppliChem, Darmstadt, Germany) for $1 \mathrm{~h}$ at room temperature, the sample was washed with PBSTx and then treated with the first antibody. One spot on the slide was incubated with rabbit anti-murine iNOS (polyclonal rabbit serum, Abcam, Cambridge, UK) and goat anti-murine Ym1 (polyclonal goat serum, R\&D Systems, Abingdon, UK; 1:50) in 10\% normal donkey serum with PBSTx and the other spot was incubated as negative control with normal rabbit and normal goat serum $(1: 80,000)$ in $10 \%$ normal donkey serum with PBSTx for $1 \mathrm{~h}$ at room temperature. After washing the spots were incubated with donkey anti-goat IgG Cy3 (polyclonal donkey serum, Dianova, Hamburg, Germany) and donkey anti-rabbit IgG Cy2 (polyclonal donkey serum, Dianova; 1:100) in 10\% normal donkey serum with PBSTx for $1 \mathrm{~h}$ at room temperature. The slide was counterstained with Hoechst 33342 at room temperature for 5 min in PBSTx, washed, and sealed with Aqua Poly/Mount (Polysciences, Warrington, PA) and a cover slip. After incubation for $1 \mathrm{~h}$ at $4{ }^{\circ} \mathrm{C}$, in minimum, the slides were analyzed with a fluorescence microscope (Olympus BX40, Olympus, Hamburg, Germany) with a XM10 camera (Olympus) and the CellSens Dimension software version 1.3 (Olympus). Per spot, at minimum, 10 high-power fields at a original magnification of $\times 400$ were photographed with a 12-bit camera (Hoechst 33342, Cy2, Cy3). All samples were treated in the same manner, the exposure time for Hoechst 33342 was $200 \mathrm{~ms}$, for Cy2 $2 \mathrm{~s}$ and for Cy3 $1 \mathrm{~s}$. The micrographs were saved in TIF format. After that the files were imported into ImageJ software (NIH, Bethesda, MD) version $1.48 \mathrm{v}$ and the gray values for the cell somas were determined by marking it (except the nucleus) and measuring the mean gray value ( 0 black and 4,095 white) of each cell. Per genotype and time point $100-400$ cells were analyzed.

Flow cytometry and cell sorting. eFluor 506 or eFluor 780 viability dye (eBioscience, Frankfurt, Germany) were used to ensure discrimination and exclusion of dead cells during analysis. For surface staining, cells were incubated for $15 \mathrm{~min}$ on ice with TruStain fcX $(0.2 \mu \mathrm{g} / 1 \times$ E6 cells; clone 93, Biolegend, Fell, Germany) and rat serum (Sigma-Aldrich) to avoid unspecific binding of antibody fluorochrome conjugates. Subsequently, staining antibodies were added for a 15-min incubation period at $4{ }^{\circ} \mathrm{C}$ in the dark. For intracellular cytokine staining cells were fixed with $2 \%$ paraformaldehyde (Serva, Heidelberg, Germany) for $20 \mathrm{~min}$ on ice. Permeabilization of cells was achieved using FACS buffer (i.e., PBS containing 3\% heat-inactivated FBS and $0.1 \%$ sodium azide) containing $0.5 \%$ saponin (w/v, Serva). For staining of transcription factors Foxp3/Transcription Factor Staining Buffer Set (eBioscience) was used. After permeabilization, cells were incubated for 15 min at $4{ }^{\circ} \mathrm{C}$ with TruStain fcX (Biolegend) and rat serum (SigmaAldrich). Afterwards, staining antibodies were added for a $30 \mathrm{~min}$ incubation on ice. Sorting of viable cells was performed with a $\mathrm{BD}$ FACSAria III. For specific stainings the following antibodies were used: anti-CD4-Alexa700 (RM4-5, Biolegend), anti-IL-5-APC (TRFK5, Biolegend), anti-IL-13-PerCp-eF710 (eBio13A, eBioscience), anti-Siglec-F-Brilliant Violet 421 (E50-2440, BD Biosciences), antiCD11c-APC (N418, eBioscience), anti-ICOS Brilliant Violet 421 (C398.4A, Biolegend), anti-Gr-1 PE-Cy7 (RB6-8C5, Biolegend), 
anti-B220 PE-Cy7 (RA3-6B2, Biolegend), anti-CD3 PE-Cy7 (1452C11, eBioscience), anti-NKp46 PE-Cy7 (29A1.4, Biolegend), antiCD11c PE-Cy7 (N418, Biolegend), anti-CD11b PE-Cy7 (M1/70, eBioscience), anti-Ki67 eFluor450 (SolA15, eBioscience), anti-Foxp3 PerCp-Cy5.5 (FJK-16 s, eBioscience), anti-GATA3 eFluor660 (TWAJ, eBioscience), anti-CD90.2 Pacific Blue (30-H12, Biolegend), antiCD25 PE (PC61.5, eBioscience), anti-KLRG1 (2F1, eBioscience), and anti-T1/ST2 (IL-33R) FITC (DJ8, MD Biosciences, Zürich, Switzerland). Antibodies used for Lineage exclusion were as follows: B220, CD3, CD4, CD11b, CD11c, Gr-1, and NKp46. Appropriate isotype antibodies were from eBiosciences and Biolegend. Cells were aquired on a BD LSRFortessa using DIVA version 6.1.1, and FlowJo version $\mathrm{X}$ (Treestar, Ashland, OR) software was used for analysis. Cell numbers and frequencies were calculated based on the frequency of CD $45^{+}$ cells of single cell lung suspension that had been checked previously for viability using a Neubauer counting chamber and additionally a Life/ Dead-discriminating stain in flow cytometry. Total numbers of an individual cell population were then back-calculated from percentages of corresponding gates with respect to the gating hierarchy.

Cytokine ELISAs. Cytokine concentrations were determined by sandwich ELISA systems. IL-5 measurement was done using the mAb TRFK5 (BD Biosciences) for capturing and the biotinylated $\mathrm{mAb}$ TRFK4 (BD Biosciences) for detection. For IL-13, the Ready-SET-Go! ELISA from eBioscience and mouse DUO-SET ELISA from R\&D Systems was used. For IL-17A mouse DUO-SET ELISA from R\&D Systems was used. Biotinylated detection antibodies were incubated with peroxidase-labeled streptavidin (Southern Biotechnology Associates, Birmingham, AL).

Statistical evaluation. The one-way analysis of variance with Dunnett's multiple comparison test was performed for group/time point comparison against a control group/time point with equally distributed values with ${ }^{\star} P \leqslant 0.05 ;{ }^{\star *} P \leqslant 0.01 ;{ }^{\star *} P \leqslant 0.001$. The oneway analysis of variance with Bonferroni's multiple comparison test was performed for group/time point comparison with equally distributed values with ${ }^{\star} P \leqslant 0.05 ;{ }^{* *} P \leqslant 0.01 ;{ }^{* *} P \leqslant 0.001$. KruskalWallis test with Dunn's multiple comparison test was performed for group/time point comparison with unequally distributed values with ${ }^{\star} P \leqslant 0.05 ;{ }^{\star \star} P \leqslant 0.01 ;{ }^{\star *} P \leqslant 0.001$.

SUPPLEMENTARY MATERIAL is linked to the online version of the paper at http://www.nature.com/mi

\section{ACKNOWLEDGMENTS}

We thank Dr A.N.J. McKenzie for providing the $i / 17 r b x t 1 / s t 2^{-1-}$ and IL-13eGFP reporter mice. rag2 ${ }^{-1-}$, rag2/ $\gamma \mathrm{C}^{-1-}$ mice were kindly provided by $\operatorname{Dr} A$. Diefenbach. We like to thank $\operatorname{DrN}$. Schütze for critical reading of the manuscript. We are grateful to A. Grohs and S. Lehnert for superb technical assistance, and R. Voigtländer and L. Mittag for keeping the mice during the experiments. Finally, we also thank C. Westermann for counting bronchoepithelial cells allowing the calculation of the PAS score. Flow cytometry was performed at the Core Unit Flow Cytometry (CUDZ) of the College of Veterinary Medicine, University of Leipzig. This project was funded by grant PI 1066/1-1 (to D.P.) and grant MU 2283/2-1 (to U.M.) by the Deutsche Forschungsgemeinschaft (German Research Foundation).

\section{DISCLOSURE}

The authors declared no conflict of interest.

2016 Society for Mucosal Immunology

\section{REFERENCES}

1. Martinez, F.D. \& Vercelli, D. Asthma. Lancet 382, 1360-1372 (2013).

2. Fahy, J.V. Type 2 inflammation in asthma-present in most, absent in many. Nat. Rev. Immunol. 15, 57-65 (2015).
3. Wenzel, S.E. Asthma phenotypes: the evolution from clinical to molecular approaches. Nat. Med. 18, 716-725 (2012).

4. Holgate, S.T. \& Polosa, R. Treatment strategies for allergy and asthma. Nat. Rev. Immunol. 8, 218-230 (2008).

5. Lloyd, C.M. \& Hessel, E.M. Functions of T cells in asthma: more than just $\mathrm{T}(\mathrm{H}) 2$ cells. Nat. Rev. Immunol. 10, 838-848 (2010).

6. Porter, P.C. et al. Airway surface mycosis in chronic TH2-associated airway disease. J. Allergy Clin. Immunol. 134, 325-331 (2014).

7. Castanhinha, S. et al. Pediatric severe asthma with fungal sensitization is mediated by steroid-resistant IL-33. J. Allergy Clin. Immunol. 136, 312-322 (2015).

8. Becker, K.L. et al. Pattern recognition pathways leading to a Th2 cytokine bias in allergic bronchopulmonary aspergillosis patients. Clin. Exp. Allergy 45, 423-437 (2015)

9. Hansbro, P.M., Kaiko, G.E. \& Foster, P.S. Cytokine/anti-cytokine therapy novel treatments for asthma? Br. J. Pharmacol. 163, 81-95 (2011).

10. Goldman, D.L., Davis, J., Bommarito, F., Shao, X. \& Casadevall, A. Enhanced allergic inflammation and airway responsiveness in rats with chronic Cryptococcus neoformans infection: potential role for fungal pulmonary infection in the pathogenesis of asthma. J. Infect. Dis. 193, 1178-1186 (2006).

11. Muller, $U$. et al. Lack of IL-4 receptor expression on Thelper cells reduces $T$ helper 2 cell polyfunctionality and confers resistance in allergic bronchopulmonary mycosis. Mucosal. Immunol. 5, 299-310 (2012).

12. Goldman, D.L. et al. Serologic evidence for Cryptococcus neoformans infection in early childhood. Pediatrics 107, E66 (2001).

13. Goldman, D.L. et al. Increased chitinase expression and fungal-specific antibodies in the bronchoalveolar lavage fluid of asthmatic children. Clin. Exp. Allergy 42, 523-530 (2012).

14. Muller, $U$. et al. IL-13 induces disease-promoting type 2 cytokines, alternatively activated macrophages and allergic inflammation during pulmonary infection of mice with Cryptococcus neoformans. J. Immunol. 179, 5367-5377 (2007).

15. Flaczyk, A. et al. IL-33 signaling regulates innate and adaptive immunity to Cryptococcus neoformans. J. Immunol. 191, 2503-2513 (2013).

16. Stenzel, W. et al. IL-4/L-13-dependent alternative activation of macrophages but not microglial cells is associated with uncontrolled cerebral cryptococcosis. Am. J. Pathol. 174, 486-496 (2009).

17. Piehler, D. et al. T1/ST2 promotes $T$ helper 2 cell activation and polyfunctionality in bronchopulmonary mycosis. Mucosal. Immunol. 6 , 405-414 (2013).

18. Zhang, Y. et al. Robust Th1 and Th17 immunity supports pulmonary clearance but cannot prevent systemic dissemination of highly virulent Cryptococcus neoformans H99. Am. J. Pathol. 175, 2489-2500 (2009).

19. Voelz, K., Lammas, D.A. \& May, R.C. Cytokine signaling regulates the outcome of intracellular macrophage parasitism by Cryptococcus neoformans. Infect. Immun. 77, 3450-3457 (2009).

20. Muller, U. et al. A gene-dosage effect for interleukin-4 receptor alpha-chain expression has an impact on Th2-mediated allergic inflammation during bronchopulmonary mycosis. J. Infect. Dis. 198, 1714-1721 (2008).

21. Sabo-Attwood, T. et al. Gene expression profiles reveal increased mClca3 (Gob5) expression and mucin production in a murine model of asbestosinduced fibrogenesis. Am. J. Pathol. 167, 1243-1256 (2005).

22. Neill, D.R. et al. Nuocytes represent a new innate effector leukocyte that mediates type-2 immunity. Nature 464, 1367-1370 (2010).

23. Spits, H. et al. Innate lymphoid cells-a proposal for uniform nomenclature. Nat. Rev. Immunol. 13, 145-149 (2013).

24. lijima, K. et al. IL-33 and thymic stromal lymphopoietin mediate immune pathology in response to chronic airborne allergen exposure. J. Immunol. 193, 1549-1559 (2014).

25. Bartemes, K.R. et al. IL-33-responsive lineage-CD25 + CD44hi lymphoid cells mediate innate type 2 immunity and allergic inflammation in the lungs. J. Immunol. 188, 1503-1513 (2011).

26. Angkasekwinai, P. et al. Interleukin 25 promotes the initiation of proallergic type 2 responses. J. Exp. Med. 204, 1509-1517 (2007).

27. Barlow, J.L. \& McKenzie, A.N. Nuocytes: expanding the innate cell repertoire in type-2 immunity. J. Leukoc. Biol. 90, 867-874 (2011).

28. Klein Wolterink, R.G. et al. Essential, dose-dependent role for the transcription factor Gata3 in the development of IL-5 + and IL-13+ 
type 2 innate lymphoid cells. Proc. Natl Acad. Sci. USA 110, 10240-10245 (2013).

29. Schulze, B. et al. CD4(+) FoxP3(+) regulatory T cells suppress fatal $T$ helper 2 cell immunity during pulmonary fungal infection. Eur. J. Immunol. 44, 3596-3604 (2014).

30. Vasanthakumar, A. et al. The transcriptional regulators IRF4, BATF and IL-33 orchestrate development and maintenance of adipose tissueresident regulatory T cells. Nat. Immunol. 16, 276-285 (2015).

31. Schiering, C. et al. The alarmin IL-33 promotes regulatory T-cell function in the intestine. Nature 513, 564-568 (2014).

32. Wohlfert, E.A. et al. GATA3 controls Foxp3(+) regulatory T cell fate during inflammation in mice. J. Clin. Invest. 121, 4503-4515 (2011).

33. Muller, U. et al. Abrogation of IL-4 receptor-alpha-dependent alternatively activated macrophages is sufficient to confer resistance against pulmonary cryptococcosis despite an ongoing $\mathrm{T}(\mathrm{h}) 2$ response. Int. Immunol. 25, 459-470 (2013).

34. Voelz, K., Lammas, D.A. \& May, R.C. Cytokine signaling regulates the outcome of intracellular macrophage parasitism by Cryptococcus neoformans. Infect. Immun. 77, 3450-3457 (2009).

35. Gordon, S. \& Martinez, F.O. Alternative activation of macrophages: mechanism and functions. Immunity 32, 593-604 (2010).

36. Byers, D.E. \& Holtzman, M.J. Alternatively activated macrophages and airway disease. Chest 140, 768-774 (2011).

37. Ford, A.Q. et al. Adoptive transfer of IL-4Ralpha + macrophages is sufficient to enhance eosinophilic inflammation in a mouse model of allergic lung inflammation. BMC Immunol. 13, 6 (2012).

38. Bunting, M.M. et al. Interleukin-33 drives activation of alveolar macrophages and airway inflammation in a mouse model of acute exacerbation of chronic asthma. Biomed. Res. Int. 2013, 250938 (2013).

39. Yang, Z. et al. Macrophages as IL-25/L-33-responsive cells play an important role in the induction of type 2 immunity. PLoS One 8, e59441 (2013).

40. Price, A.E. et al. Systemically dispersed innate IL-13-expressing cells in type 2 immunity. Proc. Natl Acad. Sci. USA 107, 11489-11494 (2010).

41. Moro, K. et al. Innate production of $\mathrm{T}(\mathrm{H}) 2$ cytokines by adipose tissueassociated c-Kit(+)Sca-1(+) lymphoid cells. Nature 463, 540-544 (2010).

42. Wills-Karp, M. Interleukin-13 in asthma pathogenesis. Immunol. Rev. 202, 175-190 (2004).

43. Bhakta, N.R. \& Woodruff, P.G. Human asthma phenotypes: from the clinic, to cytokines, and back again. Immunol. Rev. 242, 220-232 (2011).

44. Grunig, $G$. et al. Requirement for $\mathrm{IL}-13$ independently of $\mathrm{IL}-4$ in experimental asthma. Science 282, 2261-2263 (1998).

45. Wiesner, D.L. et al. Chitin recognition via chitotriosidase promotes pathologic type-2 helper T cell responses to cryptococcal infection. PLOS Pathog. 11, e1004701 (2015).

46. Ho, I.C., Tai, T.S. \& Pai, S.Y. GATA3 and the T-cell lineage: essential functions before and after T-helper-2-cell differentiation. Nat. Rev. Immunol. 9, 125-135 (2009).
47. Guo, L. et al. IL-1 family members and STAT activators induce cytokine production by Th2, Th17, and Th1 cells. Proc. Natl Acad. Sci. USA 106, 13463-13468 (2009).

48. Noval, R.M. et al. Regulatory T cell reprogramming toward a Th2-cell-like lineage impairs oral tolerance and promotes food allergy. Immunity $\mathbf{4 2 ,}$ 512-523 (2015).

49. Wang, Y., Su, M.A. \& Wan, Y.Y. An essential role of the transcription factor GATA-3 for the function of regulatory Tcells. Immunity 35, 337-348 (2011).

50. Hartl, D. et al. Quantitative and functional impairment of pulmonary CD4 + CD25hi regulatory T cells in pediatric asthma. J. Allergy Clin. Immunol. 119, 1258-1266 (2007).

51. Joller, N. et al. Treg cells expressing the coinhibitory molecule TIGIT selectively inhibit proinflammatory Th1 and Th17 cell responses. Immunity 40, 569-581 (2014)

52. Barlow, J.L. et al. IL-33 is more potent than IL-25 in provoking IL-13producing nuocytes (type 2 innate lymphoid cells) and airway contraction. J. Allergy Clin. Immunol. 132, 933-941 (2013).

53. Kondo, Y. et al. Administration of IL-33 induces airway hyperresponsiveness and goblet cell hyperplasia in the lungs in the absence of adaptive immune system. Int. Immunol. 20, 791-800 (2008).

54. Halim, T.Y. et al. Group 2 innate lymphoid cells are critical for the initiation of adaptive T helper 2 cell-mediated allergic lung inflammation. Immunity 40 , 425-435 (2014).

55. Chang, Y.J. et al. Innate lymphoid cells mediate influenza-induced airway hyper-reactivity independently of adaptive immunity. Nat. Immunol. 12, 631-638 (2011).

56. Mirchandani, A.S. et al. Type 2 innate lymphoid cells drive CD4 + Th2 cell responses. J. Immunol. 192, 2442-2448 (2014).

57. Liu, B., Lee, J.B., Chen, C.Y., Hershey, G.K. \& Wang, Y.H. Collaborative interactions between type 2 innate lymphoid cells and antigen-specific CD4 + Th2 cells exacerbate murine allergic airway diseases with prominent eosinophilia. J. Immunol. 194, 3583-3593 (2015).

58. Kabata, H. et al. Thymic stromal lymphopoietin induces corticosteroid resistance in natural helper cells during airway inflammation. Nat. Commun. 4, 2675 (2013).

59. Johnston, S.A. \& May, R.C. Cryptococcus interactions with macrophages: evasion and manipulation of the phagosome by a fungal pathogen. Cell Microbiol. 15, 403-411 (2013).

60. Coelho, C., Bocca, A.L. \& Casadevall, A. The intracellular life of Cryptococcus neoformans. Annu. Rev. Pathol. 9, 219-238 (2014).

61. Kurowska-Stolarska, M. et al. IL-33 amplifies the polarization of alternatively activated macrophages that contribute to airway inflammation. J. Immunol. 183, 6469-6477 (2009).

62. Besnard, A.G. et al. IL-33-mediated protection against experimental cerebral malaria is linked to induction of type 2 innate lymphoid cells, M2 macrophages and regulatory T cells. PLoS Pathog. 11, e1004607 (2015).

63. Fulkerson, P.C. et al. A central regulatory role for eosinophils and the eotaxin/CCR3 axis in chronic experimental allergic airway inflammation. Proc. Natl Acad. Sci. USA 103, 16418-16423 (2006). 